Bonn

\title{
ZUM TYPUS DES OSSETISCHEN KASUSSYSTEMS
}

Altiranisch ist das altindogermanische Kasussystem weitgehend erhalten geblieben; im Singular (Sg.) der avestischen (av.) Nominaldeklination werden noch die acht Kasus Nominativ, Akkusativ, Genetiv, Dativ, Ablativ, Lokativ, Instrumental und Vokativ unterschieden; z.B. o-Stamm ahura- 'Gott': ahurō, ahurəm, ahurahyā, ahurāi usw.; im Altpersischen ist dieses System durch den Zusammenfall von Dativ und Genetiv um einen Kasus reduziert worden ${ }^{1}$. Auch in der typologischen Anordnung der Morpheme hat das Altiranische den aus dem Indogermanischen (idg.) ererbten Status einer flektierenden Sprache bewahrt. Es ist charakterisiert durch Merkmale wie die Differenzierung von monothematischer und heteroklitischer Deklination ${ }^{2}$ (vgl. oStamm vs. $r / n$-Stamm: av. ahura- vs. hvarō 'Sonne', Genetiv xvōng), Caland-Wackernagelschen Suffixwechsel (av. dərəz-ra- 'fest' : dərəz-i-ra日a- 'festen Wagen habend') $)^{3}$, Ablaut (av. dātā 'Geber', Genetiv dā $\vartheta_{\text {rō) }}$, Formvariation ${ }^{4}$ (aav. Genetiv Sg. ahura-hyā vs. dā $\vartheta r-\bar{o}$ ) oder Autonomie des Wortes ${ }^{5}$.

$\mathrm{Zu}$ den Sprachwandelerscheinungen, die das ossetische Nomen von seiner altiranischen Vorstufe unterscheiden, gehören die Aufgabe der Genusdifferenzierung ${ }^{6}$, vor allen Dingen aber der Übergang von flektierender zu agglutinierender Nominaldeklination; im ossetischen Deklinationssystem sind die Funktionen von Numerus und Kasus, die altiranisch zusammen mit dem Genus in einem Morphem zusammenfallen, auf zwei monofunktionale Einheiten verteilt; in der formalen Anordnung folgt dabei das in Singular und Plural identische Kasusmorphem dem Pluralindikator:

1 Vgl. R.G. Kent, Old Persian: Grammar, Texts, Lexicon ( ${ }^{2}$ New Haven, Connecticut 1953) 57

2 Zur Terminologie - monothematisch entspricht V. Pisanis declinazione monotematica - vgl. J. Knobloch, Sprachwiss. Wörterbuch, Lfg. 7 (Heidelberg 1974) 527.

3 Vgl. E. Risch, Wortbildung der homerischen Sprache $\left({ }^{2}\right.$ Berlin-New York 1974) $218 \mathrm{f}$.

4 Zum Terminus Formvariation vgl. E. Lewy, Die Heimatfrage. In: KZ 58(1931) 7= Kleine Schriften (Berlin 1961) 205: "Die Bezeichnung derselben [...] inneren Form durch verschiedene äußere Formen ist ein durchgehendes Kennzeichen des idg. Sprachbaus"; vgl. z.B. "Genitivbildungen wie vir-i, cui-us, stella-rum".

5 Zur Definition dieses Begriffes vgl. G. Deeters, Revue de Kartvélologie Bedi Kartlisa 23 (1957) 14: "jedes Wort des Satzes ist selbststaendig und traegt an sich die Formantien, die seine Beziehung zu einem anderen Wort (Konkordanz) oder seine Funktion innerhalb des Satzes (Flexion) kennzeichnen."

6 Vgl. die Definition von Genus bei C.F. Hockett, A Course in Modern Linguistics ( ${ }^{7}$ New York 1964) 230: "Nouns belong to a gender; some adjectives are inflected for gender. For adjectives, then, gender is an inflectional category [...]. For nouns, the genders are rather what we shall call selective categories." 


\begin{tabular}{|c|c|c|}
\hline & Singular & Plural \\
\hline Nominativ/Akkusativ & saer 'Kopf' & $s c e r-t-c e$ \\
\hline Genetiv/Inessiv & scer-a & sar-t-a \\
\hline Dativ & sar-aen & sær-t-æn \\
\hline Allativ & $s a r-m a$ & sær-t-æm \\
\hline Ablativ/Instrumental & sar- $a j$ & sær-t-æj \\
\hline Adessiv & $s a-\partial l$ & sær-t-al \\
\hline Aequativ & scer-au & sær-t-au \\
\hline Komitativ & scer-imo & sær-t-imæ $\mathfrak{x}^{7}$ \\
\hline
\end{tabular}

Syntaktisch wird die Autonomie des Wortes durch Gruppenflexion mit der Basic Word Order Determinans vor Determinatum und dem Gebrauch von Postpositionen ersetzt ${ }^{8}$ :

Parataktisch: jae mad, joe fad ama ja xot-imae (Komitativ) 'mit seiner Mutter, seinem Vater und seiner Schwester'; hypotaktisch: Sg. ma zarond fad 'mein alter Vater', Dat. mae zœerond fadcen; Pl. nce zcerond fadceltce 'unsere alten Väter', Dat. nœe zarond fadceltcen ${ }^{9}$.

Morphonematischer Wechsel zwischen Singular und Plural ist entweder in konservativen Restbeständen ererbt (vgl. fəd: fədcel in Fußnote 9) oder durch die Wirkung einzelsprachlicher Lautgesetze neu generiert worden: vgl. fars 'Seite, Land': P1. farstoe: Ableitung auf -ag: farss-ag 'fremd', barceg 'Reiter': P1. bardžə-to' ${ }^{10}$. Es bleibt festzuhalten, daß die in phonetischen Veränderungen dieser Art zum Ausdruck gebrachten symbolischen (fars: fars-) und fusionierenden (barag: bardž 2 ) Techniken in der Regel nicht zu den Korrelationen des agglutinierenden Typus gehören ${ }^{11}$.

Konfrontiert man das altiranische mit dem ossetischen Kasussystem unter dem Gesichtspunkt der semantischen Differenzierung der Glieder des Paradigmas, so lassen sich eine Reihe von Transformationen feststellen. $\mathrm{Zu}$ diesen gehören die fünf

$7 \quad$ Zu dem unterschiedlichen Verhalten der ossetischen Pronomina vgl.: L. Zgusta, Zu den Subsystemen des Sprachsystems: Eine scheinbare Unregelmäßigkeit der ossetischen Deklination. In: Symbolae Linguisticae in honorem Georgii Kuryłowicz (Wrocław-Warszawa-Kraków 1965) 379-382.

8 Zum Terminus Gruppenflexion vgl. F.N. Finck, Die Haupttypen des Sprachbaus (Leipzig 1910) 144; 154 (griechische Stammflexion vs. arabische Wurzelflexion vs. georgische Gruppenflexion); "nicht selten sind es aber auch Gruppen von Elementen, die wie erst werdende Wörter verhältnismäßig lose miteinander verbunden erscheinen".

9 Das Element - $\propto$ l- beim Pl. von Verwandtschaftsnamen - fəd: fad $\not$ lt $c e$ 'Vater', mad: mad $c$ lt $\propto$ ' Mutter', cervad: arvadaltce 'Bruder, Verwandter' - reflektiert die alte Stammbildung -ar- (vgl. V.I. Abaev, Istoriko-etimologičeskij slovar' osetinskogo jazyka I (Moskva-Leningrad 1958) 489: II (1973) 62; 438.

10 Vgl. G.S. Achvlediani (Red.), Grammatika osetinskogo jazyka, Tom 1 (Ordžonikidze 1963) 8off.

11 Vgl. E. Sapir, Language (1921) Kapitel 6 und s. den introflexiven Typus von V. Skalička, TLP 2 (1966) $162 \mathrm{f}$. 
Merkmale, die im folgenden durch die Konfrontation mit typologischen Parallelen anderer Sprachen genauer bestimmt werden sollen:

I. Verstärkter Ausbau der Lokalkasus

II. Zusammenfall des vom Komitativ differenzierten Instrumentals mit dem Ablativ

III. a. Differenzierung von Allativ und Akkusativ;

b. Differenzierung des Akkusativs nach semantischen Merkmalen

IV. Entwicklung eines Aequativs

\section{Entwicklung eines prädikativen Dativs}

Ad. I. Dem altiranischen Lokativ-Ablativ-Allativ/Akkusativ ${ }^{12}$ stehen im Ossetischen Ablativ-Allativ-Inessiv-Adessiv gegenüber. Die funktionale Aufspaltung des älteren einheitlichen Lokativs (auf die Frage wo?) in zwei voneinander differenzierte Essive, den Inessiv (auf die Frage worin?: kcesag don a qazad 'the fish played in the water' ${ }^{13}$ ) und den Adessiv (auf die Frage worauf?: baxol abadti 'he sat on the horse'14), könnte auf den Einfluß des Ostkaukasischen zurückgehen. In den Sprachen der Ostkaukasischen Gruppen begegnen uns allerdings noch viel stärker ausgebaute lokalistische Systeme von bis zu sieben oder acht Essiven, die außerdem zum Unterschied vom Ossetischen nach dem Prinzip der sog. Serienbildung angeordnet sind: von dem obliquen Stamm lassen sich Essive ableiten, die ihrerseits die Basis bilden für zugehörige Allative und Ablative, manchmal auch für Translative (auf die Frage wohindurch?), z.B. tabassaranisch: Indefinitus भwan 'Stein', davon Obliquus (=Ergativ) Ywan-3̌i, davon Essiv (der Bedeutung unter) भwan-3̌i-kk 'unter dem Stein', davon Allativ भwan-3̌i-kk-na 'unter den Stein' und Ablativ wwan-3̌i-kk-an 'von unter dem Stein her'. Für das Südtabassaranische unterscheidet A. Magometov ${ }^{15}$ Essive der Bedeutung in, auf, hinter, unter neben, inmitten, auf vertikaler Fläche.

Ad. II. Der komplexeste unter den ossetischen Lokalkasus ist der Ablativ; er begegnet auch in instrumentaler Funktion (farcetcej amajan 'to trim with an ax' ${ }^{16}$ ), eine semantische Kombination, wie sie auch dem Italischen bekannt ist ${ }^{17}$. Andererseits erklärt $\mathrm{O}$. Tedeevi ${ }^{18}$ den Zusammenfall beider Funktionen synkretitisch durch

12 Zum Allativ vgl. av. kạ̄m zą̄m āyenī 'in welches Land soll ich gehen?' Y. 46,1 bei H. Reichelt, Awestisches Elementarbuch (Heidelberg 1909) § 436; altpersisch ašiyava Bābirum 'er ging nach Babylon' DB III 82 bei Kent, 1. c. 80 .

13 Vgl. V.I. Abaev, A Grammatical Sketch of Ossetic (The Hague 1964) 19.

14 Vgl. Abaev, 1.c.

15 Tabasaranskij jazyk (Tbilisi 1965) 117.

16 Vgl. Abaev, 1.c.

17 Vgl. M. Leumann - J.B. Hofmann - A. Szantyr, Lateinische Grammatik, 2. Band Syntax und Stilistik, 1. Lieferung (München 1963) 101: "Im lateinischen Abl. sind in uritalischer Zeit zwei Kasus zusammengeflossen, der idg. Ablativ oder Separativ ('Woher'- Kasus, Kasus der Trennung und Absonderung) und der idg. Instrumentalis ('Mit'-Kasus, Kasus der Gemeinschaft)".

18 Senišvnebi kartul-osuri enobrivi urtiertobidan. In: Jubilejnyj sbornik posvjaščennyj G.S. Achvlediani v 
Übertragung des instrumentalen Kasusmorphems auf den Ablativ nach altgeorgischem Modell. Grundsätzlich sollte jedoch beachtet werden, daß die Entscheidung über die Priorität von Ablativ oder Instrumental im Ossetischen die etymologische Identifizierung des Morphems - $2 j$ voraussetzt ${ }^{19}$. Unabhängig von der Klärung dieses Problems dürfte der Zusammenfall beider Kasus im Ossetischen von der Spaltung des im Altiranischen komplexen (d.h. den Komitativ einschließenden) Instrumentals begleitet sein:

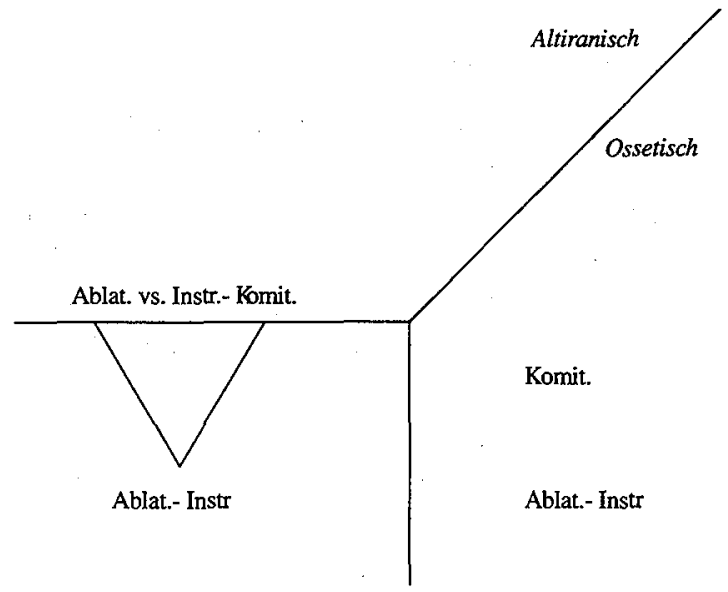

Auf komplexen, den Komitativ einschließenden, Instrumental weisen könnte noch die dialektisch unterschiedliche Wiedergabe des Komitativs im Ossetischen: das in seinen Merkmalen in der Regel archaischere Digorische bedient sich dafür des Genetivs in Verbindung mit der Postposition xaccae 'zusammen mit' (z.B. cenbali xaecce 'zusammen mit dem Genossen') ${ }^{20}$, während das Ironische ein eigenes Kasusformans -imoe entwickelt hat. Eine typologische Parallele für die uneinheitliche Wiedergabe des Komitativs findet sich im Tocharischen, wo die Präposition A śla, B śle in Verbindung mit dem Obliquus als vermutlich ältere Variante anstelle des Komitativs auf A -aśšäl, B -mpa begegnet: A ākläslyesaśśäl syak metär = B aklaṣlyemmpa ṣesa maitare 'Sie gingen zusammen mit den Schülern'21.

Ad. III. Der Allativ, zu dessen Funktionen auch die eines Finalkasus gehört (čndz donma acəd 'the daughter-in-law went for water'), fällt nicht mit dem Akkusativ als Objektkasus eines transitiven Verbums zusammen. Letzterer begegnet in zwei Varianten: in Verbindung mit 'indefinite or impersonal things' (Abaev 1964, 17) bleibt

svjazi s 80-letiem so dnja roždenija (Tbilisi 1969) 369-374.

19 Vgl. dazu V. Miller, Jazyk osetin (Moskva-Leningrad 1962) 78f.

20 Vgl. M.I. Isaev, Digorskij dialekt osetinskogo jazyka: Fonetika, morfologija (Moskva 1966) 108.

21 Vgl. W. Krause-W. Thomas, Tocharisches Elementarbuch. Bd. 1. Grammatik (Heidelberg 1960) 86, wo auf die Möglichkeit hybrider Bildungen bei Gruppenflexion hingewiesen wird: A śla niṣpal yătluneyaśśäl 'mit Besitz (und) mit Fähigkeit'. 
er (wie der Nominativ) unmarkiert; in Verbindung mit 'definite and personal beings' (Abaev 1. c. 18) wird ihm dagegen das Suffix - $\underline{a}$ angefügt, das unabhängig davon auch den Inessiv charakterisiert. Für beide Merkmale - (a) Differenzierung von Allativ und Aklkusativ, (b) Markierung des akkusativischen Zielkasus in Abhängigkeit von den semantischen Merkmalen des Nomens-liegen typologische Parallelen vor.

Ad. a. Eine Parallele für die Spaltung des idg. Akkusativs/Allativs bietet das Tocharische. Zum Unterschied von dem auf den Akkusativ zurückgehenden Obliquus, der gewissermaßen po principu dvuch osnov ${ }^{22}$ als Order 1 (= Position 1$)^{23}$ die Basis bildet für die sekundären Kasus, gehört der Allativ auf A - $a c, \mathrm{~B}-\dot{s}(c)$ der Gruppe ebendieser durch Affixe markierten sekundären Kasus an. Nach den Feststellungen von W. Thomas ${ }^{24}$ begegnet der unmarkierte Obliquus der Richtung noch bei Verben der Bewegung, "wenn eine allgemeine Richtungsangabe gemeint ist" oder bei festen Verbindungen (1. c. 42), während der Allativ - z.T. in Konkurrenz zu Lokativ und Perlativ - stärkere Präzisierung und Bedeutungsdifferenzierung ermöglicht (1. c. 44), z.b. (1. c. 25) Obliquus: A oșke, B oskai 'nach Hause' (allgemein) gegenüber Allativ: B ājīvikemts cemts akalșlyepi masa ostaś 'Er ging zum Hause eines Schülers dieser Ajīvikas' (präzise Ortsangabe). Die kontextbedingte Zurückdrängung des auf den idg. Akkusativ/Allativ zurückgehenden tocharischen Obliquus ${ }^{25}$ durch markierte Richtungskasus, darunter besonders durch den Allativ als Neubildung, entspricht der in idg. Sprachen weit verbreiteten Tendenz zur zusätzlichen Markierung des ererbten Allativs auf $/ \mathrm{m} /$ durch Präpositionen/Postpositionen: Im Griechischen wird der als Archaismus für das Epos und die attische Tragödie nachgewiesene bloße Allativ/Ak-

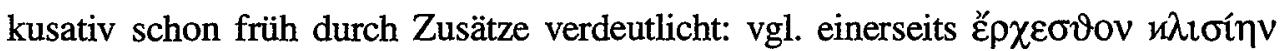
$\Pi \eta \lambda \eta \imath \alpha \delta \delta \omega$ 'A $\chi \imath \lambda \tilde{\eta} \circ \varsigma_{\text {A }} 332$ “Gehet beide zum Zelte des Peleiaden Achilleus”, an-

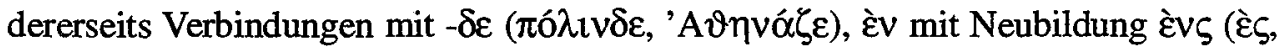
$\varepsilon i \varsigma$ ), $\pi \rho o ́ s$ usw. Die Wendungen $i$ malam crucem (neben $i$ in malam crucem) und proficisci exilium sind auf das Altlateinische beschränkt usw. ${ }^{26}$

Ad.b. Die Markierung des Zielkasus von Personen entspricht einer weit verbreiteten Tendenz, die für das Protoidg. mit seiner Differenzierung von genus commune (masculinum und femininum) vs. genus neutrum bereits von A. Meillet ${ }^{27}$ auf eine

22 Vgl. die Definition bei Magometov, 1.c. 97: “ot imenitel'nogo padeža (padeža samogo po sebe neoformlennogo, [...]) obrazuetsja ergativ, a ergativ ležit v osnove kosvennych padežej".

23 Vgl. Verf., Agglutination und Postposition im Tocharischen: MSS 25 (1969) 105-1 12.

24 W. Thomas, Der tocharische Obliquus im Sinne eines Akkusativs der Richtung: AAWL 1983 Nr. 6 (Wiesbaden 1983) 9.

25 Allerdings war im Protoidg. als einer Aktivsprache zunächst nur der Allativ als Richtungskasus durch die Postposition $/ \mathrm{m} /$ markiert; die Übernahme dieses Morphems durch den unmarkierten Zielkasus war ein späterer Prozess (s. auch im folgenden unter IIIlb).

26 Vgl. K. Brugmann/B. Delbrück, GrundriB der vergleichenden Grammatik der idg. Sprachen II $2 / 1$ und 2 ( ${ }^{2}$ Straßsurg 1911; Nachdruck Berlin 1967) 627ff.; E. Schwyzer, Griech. Gramm. II (München 1950) 67f.; E. Leumann/J.B. Hofmann/A. Szantyr, Lat. Gramm. II (München 1965) 49f. u.a. 
Formel gebracht worden war: "Le trait essentiel de la distinction de l'animé et de l'inanimé en indo-européen consiste dans la charactéristique de l'accusatif animé, au singulier et au pluriel.' Das durch spätere Entwicklungen im Romanischen, Tocharischen, Slavischen und Neuarmenischen wiederholte Phänomen ${ }^{28}$ wurde von F. Vil$\operatorname{lar}^{29}$ auf der Basis einer Belebtheitshierarchie systematisch behandelt; es hat seine Parallelen auch in nichtidg. Sprachen, für die man das Prinzip aufgestellt hat "that separate accusative marking and verb object agreement are more likely with noun phrases that are high in animacy or definiteness"30. Auf Ergativsprachen angewandt, impliziert dieser Grundsatz die These von M. Silverstein"31: "If an ergative system splits simply into two two-way case-marking schemes, then minimally either the $[+$ ego $]$ or the $[+\mathrm{tu}]$ forms are nominative-accusative, the rest ergative-absolutive." 32

Ad. IV. Der von É. Benveniste ${ }^{33}$ similatif genannte Aequativ geht auf den prädikativen Gebrauch von Bildungen auf /aul zurück: fatau ataxti 'he flew like an arrow'; čazgaen ja casgom xurau ruxs uədi 'the girl's face shone like the sun' (Abaev 1964, 19). An Adjektive gefügt, dient das Morphem /au/ der Ableitung von Adverbia: xorz 'gut' (Adj. + Adverb): xorzau (Adverb), iron 'ossetisch': ironau (Abaev 1964, 31; Benveniste, 1. c.). Die Kategorie des Aequativs ist auch aus den keltischen Sprachen bekannt ${ }^{34}$. Ihre mit dem Suffix -ithir, -idir gebildete altirische Variante kann jedoch nur von Adjektiven abgeleitet werden ${ }^{35}$ und fungiert neben Komparativ und Superlativ als Komparationsgrad: dían 'schnell', Kompar. déniu, Aequativ dénithir 'so schnell wie', trén 'stark', Kompar. tressa, Superl. tressam, Aequat. tresithir ${ }^{36}$. Das von dem

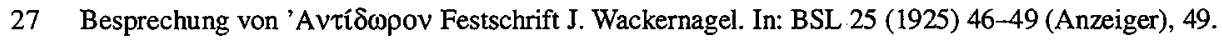

28 Im Ostarmenischen ist der Akkusativ dem Dativ gleich "bei Substantiven, die ein vernünftiges Wesen bezeichnen und zugleich auch bestimmt sind" (vgl. A. Abeghian, Neuarmenische Grammatik, Berlin und Leipzig 1936, 63).

29 F.V., Ergatividad, acusatividad y genero en la familia lingüistica indoeuropea (Salamanca 1983) 191ff.; vgl. dazu die Besprechungen von R. Ködderitzsch, Kratylos 30 (1985) 78ff. und Verf., IF 91 (1986) 344ff.; vgl. auch F.V., Ergativity and animate/inanimate gender in Indo-European: KZ 97 (1984) 167-196.

30 B. Comrie, Language Universals and Linguistic Typology (Oxford 1981) 212.

31 Hierarchy of Features and Ergativity. In: R.M.W. Dixon (ed.), Grammatical Categories in Australian Languages (Canberra 1976) 112-171, 122

32 Vgl. auch die Sprachen Eastern Pomo und Guaraní: S. McLendon, Ergativity, Case and Transitivity in Eastern Pomo. In: IJAL 44 (1978) 1-9, 6 "that the system splits common nouns and personal nouns for which agent function must be marked, from pronouns, kinship terms and proper names for which patient function must be marked"; G. Bossong, Syntax und Semantik der Fundamentalrelation. Das Guaraní als Sprache des aktiven Typs. In: Lingua 50 (1980) 359-379: bei belebten Wesen wird der Zielkasus durch die Postposition - pe markiert; G. Mallinson/B.J. Blake, Language Typology. Cross-linguistic Studies in Syntax (Amsterdam-New York-Oxford 1981) 52.

33 Études sur la langue ossète (Paris 1959) 104.

34 Vgl. W. Meid, Zum Aequativ der keltischen Sprachen, besonders des Irischen. In: W. Meid (Hrsg.): Beiträge zur Indogermanistik und Keltologie, J. Pokorny zum 80. Geburtstag gewidmet (Innsbruck 1967) 223-242.

35 Das gilt nicht für die britannischen Formen mit Präfix *kom-, die auch mit Substantiva komponiert werden: kymrisch cyf-liw 'von gleicher Farbe', cyf-led 'von gleicher Breite' (Meid, 1. c. 224). 
Aequativ abhängige Nomen steht entweder (a) im Akkusativ oder wird (b) durch einen konjunktionslosen Satz angeschlossen: (a) sonartaidir slébe 'as strong as mountains' (Thurneysen, 1. c. 157), (b) is firithir ad.fiadar 'it is as true (as) is reported' (Thurneysen, 1.c.).

Ad. V. Der Terminus datel'nyj predikativnyj 'prädikativer Dativ' wurde in die ossetische Syntax eingeführt von G. Achvlediani ${ }^{37}$, der die damit benannte Konstruktion sowohl vom dativus finalis als auch vom prädikativen Nominativ (bzw. Akkusativ) abgrenzt und mit dem prädikativen Instrumental des Baltischen und Slavischen vergleicht: Der prädikative Nominativ (resp. Akkusativ) macht eine essentielle, der prädikative Dativ eine akzidentielle Aussage: Asana fədgcenceg (Nom.) uədi = 'Asana byl pritesnitel' vs. uəj nce ronaen (Dat.) kuə uaid = 'to bylo by nam pojasom (Instr.) (1. c. 28) ${ }^{38}$. Im Ossetischen haben (a) die mit dem Adjektiv im Instrumental/Ablativ verbundenen Verben volle semantische Eigenbedeutung - Achvlediani bezeichnet sie als polnoznamenatel'nye glagoly -, während (b) die mit dem Substantiv im prädikativen Dativ konstruierten Verben, den russischen Wendungen mit prädikativem Instrumental vergleichbar, über weniger autosemantische Merkmale verfügen und deshalb von Achvlediani poluznamenatel'nye glagoly genannt werden. Wie Achvlediani 1. c. 33 weiter ausführt, verstärkt in der Gruppe (a) das Adjektiv im Instrumental die lexikalische Bedeutung des Verbums, während in der Gruppe (b) das Substantiv im Dativ semantisch weitgehend unabhängig bleibt: (a) fastcma nart ank'ardcej (Instr.) razdoextəstə = nazad narty ogorčennye (pečal'no) vorotilis' vs. (b) čcemcen razəna aj fartan (Dat.) ruxsə cemae bəndurcen (Dat.) fasdagon (= aenuson) xcersdə= daby javitsja synom (Instr.) sveta i naslednikom (Instr.) večnych blag.

Wenn man die Entwicklung des prädikativen Dativs zur Bezeichnung eines akzidentellen Zustandes als Neuerung des Ossetischen wertet, so bietet sich die in der späteren irischen Sprachentwicklung aufgekommene Konstruktion des Typus tá sé ' $n$ - $a$ rígh 'er ist König', wörtlich 'er ist in seinem König', als typologische Parallele $a^{39}$.

In altirischer Zeit verfügte die Sprache lediglich über den appositiven Dativ, der infolge von Kasussynkretismus älterem Instrumental entsprach: $a$ triur 'all three', wörtlich: 'in their three-men', isníni firiónaib 'we being righteous'. Die Konstruktion des Typus tá sé ' $n-a$ rígh mit Possessivpronomen und Präposition in begegnet im

36 Vgl. R. Thurneysen, A Grammar of Old Irish. Revised and Enlarged Edition (Dublin 1946) $232 \mathrm{ff}$.

37 G. Achvlediani, Datel'nyj predikativnyj v osetinskom jazyke. In: Vestnik Tbilisskogo gosudarstvennogo universiteta III (1923) 129-140 = Sbornik izbrannych rabot po osetinskomu jazyku I(Tbilisi 1960) 26-42.

38 Vgl. Brugmann/Delbrück, Grundriß II $2 / 1$ u. $2\left({ }^{2}\right.$ Straßburg 1911) 537ff., die von einem prädikativen Instrumental sprechen, der 'im Baltisch-Slavischen' auch 'als prädikative Apposition zu beliebigen Substantiva des Satzes' verwendet wird.

39 Vgl. dazu H. Pedersen, Tá sé 'n-a rígh: ZCP 2 (1899) 377-381; J. Baudiš, Nominalsätze und Nominalprädikate im Irischen: ZCP 9 (1913) 309-335; M. Dillon, Nominal Predicates in Irish: ZCP 16 (1927) 313-356, ZCP 17 (1928) 307-346; H. Lewis/H. Pedersen, A Concise Comparative Celtic Grammar ( ${ }^{3}$ Göttingen 1974) 163f. 
Mittelirischen zunächst als Apposition: is bés dúibsi in-far-n-ultaib LL 112 b 47 'es ist Sitte für Euch Ulsterleute' (Aedersen 1899, 379), wörtlich 'es ist Sitte für Euch in Eurem Ulsterleute'. Die nicht vor 1100 nachweisbaren prädikativen Konstruktionen des Typus tá sé 'na rígh, tá sé i $n$-a fhear 'er ist ein Mann' mit dem Verbum tá als Prädikat unterscheiden sich von dem Typus is fer-som, später is fear é 'er ist ein Mann' mit Kopula is und Prädikatsnomen im Nominativ als Prädikat semantisch dadurch "that the former describes a condition or state while the latter is a mere definition" (Dillon 1927, 316). Wenn auch die diachronen Entwicklungen im Ossetischen und Irischen ganz unterschiedlich verlaufen sind, so bleiben doch die Ergebnisse vergleichbar: jüngere, auf Substantive begrenzte, Substitute zur Bezeichnung eines akzidentiellen Zustandes (der prädikative Dativ des Ossetischen, die tá sé 'n-a rígh-Konstruktion des Irischen) führen zur Begrenzung bzw. Verdrängung des ererbten prädikativen Instrumentals.

\section{Povzetek \\ OSETSKI SESTAV SKLONOV TIPOLOSKKO}

A. Staroiranske in osetske sklonske oblike protistavno.

Staroiranske poteze: fleksijskost, razlikovanje med enotematsko in heteroklitično sklanjatvijo, menjava pripon po Calandu in Wackernaglu, oblikovne dvojnice, samostojnost besede.

Osetske poteze: aglutinativnost, opust vplivajočega spola, sklanjanje cele besedne zveze hkrati, osnovni besedni red je določujoče pred določenim, postpozicije. Aglutinativnosti nasprotne morfonematske menjave so bodisi podedovane $v$ ostankih bodisi nastale na novo zaradi delovanja poznejših glasovnih zakonov.

B. Tipoloska vzporeditev petih nadaljnjih potez osetskega sklonskega sestava $z$ drugimi jeziki:

I. Obilnejši razvoj prostorskih sklonov: tipološka vzporeditev $s$ t.i. serijsko tvorbo v vzhodnokavkaškem jeziku tabasaranščini.

II. Sovpad ablativa $z$ orodnikom, ki je nastal iz komitativa: tipološka vzporeditev s staro gruzinščino (kjer pa se ablativ in orodnik ne razlikujeta); neenako vedênje ligurskih in ironskih narečij ima vzporednosti $v$ toharščini.

III. a. Razlikovanje med alativom in tožilnikom: vzporednosti v toharščini, grščni, latinščini itd. b. Delitev tožilnika po pomenskih potezah: vzporednosti v praindoevropščini in v neindoevropskih jezikih.

IV. Ekvativ: vzporeditev $z$ razmerami v stari irščini.

V. Predikativni (= povedkovodoločilni) dajalnik rabi - primerljivo predikativnemu orodniku $\mathrm{v}$ baltš̌čni in slovanščini - kot izjava o nebistveni (priložnostni) lastnosti; $v$ tem je nasproten predikativnemu imenovalniku (oz. tožilniku) kot izjavi o bistveni lastnosti; take razmere se dajo primerjati z irskimi zgradbami. $V$ irščini je dalje tako, da rabi verbum substantivum ob priložnostni lastnosti, kopula $(=$ vez) pa ob bistveni. 